\title{
CIPIONATO DE ESTRADIOL E BENZOATO DE ESTRADIOL EM PROTOCOLOS DE INSEMINAÇÃO ARTIFICIAL EM TEMPO FIXO EM NOVILHAS MESTIÇAS
}

\author{
Roberto Ramos Sobreira ${ }^{1}$ \\ Italo Câmara de Almeida \\ Fabricio Albani Oliveira \\ Jeanne Broch Siqueira \\ Graziela Barioni \\ Diogo Vivacqua de Lima \\ Larissa Ataide Siqueira
}

\begin{abstract}
RESUMO
Avaliou-se a eficiência de dois indutores da ovulação: cipionato de estradiol (CE) e benzoato de estradiol (BE), na inseminação artificial em tempo fixo (IATF). Foram utilizadas 128 novilhas. Todos os animais foram submetidos ao mesmo protocolo de sincronização, a partir do dia 8 os animais foram distribuídos em dois tratamentos: Tratamento com CE no dia 8 (64 novilhas) e tratamento com BE no dia 9 (64 novilhas). Quanto ao número de folículos, só observou-se diferença significativa entre os tratamentos no dia 8. Não houve diferença ( $>>0,05)$ para taxa de crescimento folicular, diâmetro do folículo dominante $(10,97 \pm 1,24 \mathrm{e}$ $9,77 \pm 1,36 \mathrm{~mm})$, e intervalo da ovulação à inseminação artificial $(13,20 \pm 3,79$ e 23,00 \pm 14,28 horas) entre os tratamentos. A retirada do dispositivo de progesterona à ovulação, em

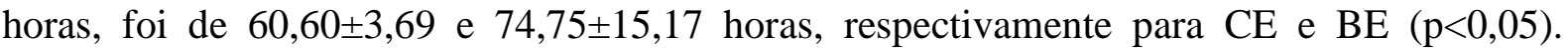
Houve diferença $(p<0,05)$ entre os tratamentos para a taxa de ovulação, que foi de $90,00 \%$ e $40,00 \%$ para CE e BE, respectivamente. A taxa de prenhez foi de $37,3 \%(p>0,05)$ para ambos tratamentos. A administração de CE no dia 8 possibilitou a redução de um manejo na IATF em novilhas sem influenciar na taxa de prenhez.
\end{abstract}

Palavras-chave: hormônios, indutores de ovulação, fisiologia reprodutiva, manejo, novilhas.

\section{ESTRADIOL CYPIONATE AND ESTRADIOL BENZOATE IN ARTIFICIAL INSEMINATION PROTOCOLS IN FIXED TIME IN HEIFERS CROSSBRED}

\begin{abstract}
We evaluated the efficiency of two ovulation induction: estradiol cypionate (EC) and estradiol benzoate (EB), at fixed time artificial insemination (TAI). 128 heifers were used. All animals were subjected to the same synchronization protocol, from the 8th day animals were divided into two treatments: Treatment with CE on the 8th (64 heifers) and treatment with EB on Day 9 (64 heifers). Regarding the number of follicles, only there was a significant difference between treatments at day 8 . There was no difference $(p>0.05)$ for follicular growth rate, diameter of the dominant follicle $(10.97 \pm 1.24$ and $9,77 \pm 1.36 \mathrm{~mm})$ and range of ovulation in artificial insemination $(13.20 \pm 3.79$ and $23.00 \pm 14.28$ hours $)$ between treatments. The withdrawal of progesterone device ovulation in hours was $60.60 \pm 3.69$ and $74.75 \pm 15,17$ hours respectively for $\mathrm{CE}$ and $\mathrm{BE}(\mathrm{p}<0.05)$. There were differences $(p<0.05)$ between treatments for ovulation rate, which was $90.00 \%$ and $40.00 \%$ for CE and BE, respectively. The pregnancy rate was $37.3 \%$ (p 0.05 ) for both treatments. The EC administration on 8
\end{abstract}

${ }^{1}$ Centro de Ciências Agrárias da Universidade Federal do Espírito Santo. Contato correspondência.

Sobreira RR, Almeida IC, Oliveira FA, Siqueira JB, Barioni G, Lima DV, Siqueira LA. Cipionato de Estradiol e Benzoato de Estradiol em protocolos de inseminação artificial em tempo fixo em novilhas mestiças. Vet. e Zootec. 2017 Set.; 24(3): 581-591. 
enabled the reduction of management in the TAI in heifers without influencing pregnancy rate.

Keywords: animal breeding, hormones, ovulation-inducing, reproductive physiology, management, heifers.

\title{
CIPIONATO DE ESTRADIOL Y BENZOATO DE ESTRADIOL EN LOS PROTOCOLOS DE INSEMINACIÓN ARTIFICIAL EN TIEMPO FIJO EN VAQUILLAS MESTIZAS
}

\begin{abstract}
RESUMEN
Se evaluó la eficiencia de la inducción de la ovulación de dos: cipionato de estradiol (CE) y benzoato de estradiol (EB), en la inseminación artificial de tiempo fijo (TAI). Se utilizaron 128 novillas. Todos los animales fueron sometidos al mismo protocolo de sincronización, a partir de los animales se dividieron en dos tratamientos $8^{\circ}$ : El tratamiento con CE en la octava (64 novillas) y el tratamiento con EB en el Día 9 (64 novillas). En cuanto al número de folículos, solamente había una diferencia significativa entre los tratamientos en el día 8. No hubo diferencia $(\mathrm{p}>0,05)$ para la tasa de crecimiento folicular, el diámetro del folículo dominante $(10,97 \pm 1,24$ y $9,77 \pm 1,36 \mathrm{~mm})$ y el rango de la ovulación en la inseminación artificial $(13,20 \pm 3,79$ y $23,00 \pm 14,28$ horas $)$ entre tratamientos. La retirada del dispositivo de progesterona ovulación en horas fue 60,60 \pm 3,69 y 74,75 \pm 15,17 horas, respectivamente, para el CE y BE $(\mathrm{p}<0,05)$. Hubo diferencias $(\mathrm{p}<0,05)$ entre los tratamientos para la tasa de ovulación, que fue $90,00 \%$ y $40,00 \%$ para CE y ser, respectivamente. La tasa de embarazo fue de 37,3\% (p 0,05) en ambos tratamientos. La administración CE el 8 de activar la reducción de la gestión en el TAI en novillas sin influir en la tasa de embarazo.
\end{abstract}

Palabras clave: las hormonas, los inductores de la ovulación, fisiología reproductiva, gestión, vaquillas.

\section{INTRODUÇÃO}

O estradiol é um hormônio esteróide, ou seja, faz parte de um grupo de lipídeos e está distribuído por todo o organismo. Em bovinos e em outras espécies é sintetizado principalmente nos folículos dominantes nos ovários, mas outros tecidos também podem produzi-lo, como por exemplo, a placenta, o córtex adrenal, testículos, cérebro e outros (1).

Os estrógenos possuem diversas funções na fisiologia reprodutiva como expressão do estro, características sexuais secundárias, indução da liberação do hormônio liberador de gonadotrofinas (GnRH) e do hormônio luteinizante (LH) (2). O estradiol e seus ésteres, como são chamados os compostos, são frequentemente empregados para promover o controle farmacológico do ciclo estral, uma vez que na ausência de progesterona sua administração pode estimular a liberação de GnRH e LH e causar a ovulação do folículo dominante (3).

Esses estrógenos produzem diferentes perfis de circulação de estradiol, provavelmente devido diferenças na esterificação da molécula, o que leva a alterações na polaridade e no peso molecular das estruturas e, portanto, modifica a absorção e o metabolismo no organismo (4). O benzoato de estradiol tem sido largamente utilizado como indutor da ovulação quando administrado $24 \mathrm{~h}$ após a retirada da progesterona, entretanto, tem sido avaliada sua substituição por um análogo hormonal, denominado cipionato de estradiol, aplicado concomitante a retirada da progesterona $(3,5)$. 
O cipionato de estradiol (CE), como indutor da ovulação, é uma alternativa que possibilita a realização de protocolos de IATF com apenas três períodos de manejo, reduzindo a mão de obra e minimizando o estresse do animal. Permanecendo biodisponível de forma sistêmica por maior tempo, o CE administrado juntamente com a retirada do implante de progesterona, mantêm níveis plasmáticos suficientes para estimular o pico de LH após a queda dos níveis de progesterona $\left(\mathrm{P}_{4}\right)(6)$.

Programas de sincronização e indução da ovulação já têm sido utilizados com frequência em novilhas Bos taurus taurus (7) e em novilhas Bos taurus indicus $(8,9)$. No entanto em novilhas mestiças (Bos taurus indicus x Bos taurus taurus) os trabalhos são reduzidos e inconsistentes.

Assim, objetivou-se com este estudo, avaliar a utilização do cipionato de estradiol e benzoato de estradiol em protocolos de IATF em novilhas mestiças Bos taurus indicus x Bos taurus taurus, quanto à dinâmica folicular e a taxa de prenhez.

\section{MATERIAL E MÉTODOS}

O experimento foi realizado em uma propriedade localizada no Município de Dores do Rio Preto, no estado do Espírito Santo, situado nas coordenadas geográficas 20 41' Sul, $41^{\circ} 50^{\prime}$ Oeste e a $774 \mathrm{~m}$ de altitude, no período de fevereiro a junho de 2014 . O clima predominante da região é o tropical com verões quentes e chuvosos e invernos frios e secos, temperatura média anual de $19,2^{\circ} \mathrm{C}$ e precipitação pluviométrica de $1.730 \mathrm{~mm}$ anuais.

Foram utilizadas, 128 novilhas mestiças (Bos taurus indicus x Bos taurus taurus), com escore de condição corporal (ECC) acima de 2,5, que apresentavam, ausência de sinais clínicos de doença infecciosa ou metabólica, e de alterações dos órgãos genitais ao exame ginecológico.

O manejo das novilhas foi em regime extensivo, utilizando-se a pastagem composta principalmente de braquiarão (Brachiaria brizantha cv. Marandu). Sal proteinado, mineral e a água foram fornecidos ad libitum.

Todas as novilhas foram submetidas a um protocolo de sincronização: no dia 0 (D0), inserção de dispositivo intravaginal de progesterona (DIV) e aplicação intramuscular (IM) de $2 \mathrm{mg}$ de benzoato de estradiol (BE); dia 8 (D8): retirada do DIV, aplicação IM, de 0,15mg de cloprostenol sódico $\left(\mathrm{PGF}_{2 \alpha}\right)$ e aplicação IM de $300 \mathrm{UI}$ de eCG. No dia 8 os animais foram distribuídos aleatoriamente em dois tratamentos: 1) Tratamento com cipionato de estradiol (CE) no dia 8 (64 animais) - aplicação de 1mg de CE por via IM, no D8 (TCE8). 2) Tratamento com BE no dia 9 (64 animais) - aplicação de $1 \mathrm{mg}$ de BE no D9 por via IM (TBE9). Foram inseridos em todas as novilhas implantes de $\mathrm{P}_{4}$ de $2^{0}$ ou $3^{0}$ uso, de forma aleatória. Todos os manejos de aplicação dos fármacos foram entre 09:00 e 10 horas da manhã, dos respectivos dias.

Em cada grupo, dez animais foram separados para avaliação da dinâmica folicular. Os exames ultrassonográficos destas 20 novilhas foram realizados nos dias 0,8 e no dia 10 . A partir do D10 a US foi realizada de 12 em 12 horas até a determinação da ovulação pela ausência do folículo dominante, com aparelho portátil de ultrassom acoplado a um transdutor linear retal de 5,0 MHz (MINDRAY ${ }^{\circledR}$, modelo DP2200 VET). Assim, foi verificado no D0 o status folicular, e nos demais dias avaliadas a dinâmica folicular.

Os folículos foram classificados de acordo com o diâmetro em pequenos ( $\mathrm{FP}<6 \mathrm{~mm})$, médios (FM de 6 a $8 \mathrm{~mm}$ ) e dominantes (FD $>8 \mathrm{~mm}$ ) (10). Foi calculada a taxa de crescimento folicular (mm/dia), pela divisão da diferença do tamanho do folículo dominante do dia 10 pelo do dia 8, dividido pelo número de dias (D10-D8/2) e o diâmetro do folículo dominante. Estabeleceu-se também o tempo (em horas) da retirada do dispositivo de progesterona à

Sobreira RR, Almeida IC, Oliveira FA, Siqueira JB, Barioni G, Lima DV, Siqueira LA. Cipionato de Estradiol e Benzoato de Estradiol em protocolos de inseminação artificial em tempo fixo em novilhas mestiças. Vet. e Zootec. 2017 Set.; 24(3): 581-591. 
ovulação, da IA à ovulação e a taxa de ovulação (números de animais que ovularam de cada tratamento, dividido pelos animais dos respectivos tratamentos).

As inseminações artificiais (IA) foram realizadas 48 e $52 \mathrm{~h}$ após a retirada do dispositivo intravaginal de $\mathrm{P}_{4}$ para os tratamentos com CE e BE respectivamente, sendo feitas pelo mesmo técnico, utilizando sêmen convencional de touros da raça nelore, gir e holandês, de acordo com as características raciais de cada novilha, de uma central de sêmen associada à Associação Brasileira de Inseminação Artificial (ASBIA). No $30^{\circ}$ dia após a IATF, os animais foram avaliados por meio de ultrassonografia, e a taxa de prenhez foi determinada em cada tratamento.

A análise estatística foi realizada utilizando o programa SAS Studio (11), em delineamento inteiramente casualizado, considerando os efeitos dos tratamentos. As variáveis com distribuição normal, como o diâmetro do folículo dominante e taxa de crescimento folicular foram analisadas utilizando-se o teste de $t$ independente. Quanto aos dados não paramétricos como o número de folículos $<6 \mathrm{~mm}, 6-8 \mathrm{~mm}$ e $>8 \mathrm{~mm}$, intervalo da retirada do implante de $\mathrm{P}_{4}$ à ovulação e da IATF à ovulação, que apresentaram distribuição não normal, foram avaliadas mediante teste de Mann-Whitney.

Os dados de taxa de ovulação e gestação foram organizados em tabelas de contingência e analisados pelo teste de Qui-quadrado $\left(\chi^{2}\right)$. O nível de significância adotado foi $\alpha=0,05$.

\section{RESULTADOS E DISCUSSÃO}

Quanto ao número de folículos, classificados de acordo com seu tamanho em folículos $<6 \mathrm{~mm}$, 6-8mm e > 8mm, não houve diferença $(\mathrm{p}>0,05)$ entre os tratamentos nos dias 0,8 e 10 para os folículos pequenos e médios e, para os folículos $>8 \mathrm{~mm}$ foi observada diferença $(\mathrm{p}<0,05)$ entre tratamentos no D8 (Tabela 1). Dos 10 animais submetidos ao TBE9 seis não ovularam ao final do tratamento. Do D0 ao D8 o protocolo hormonal administrado aos animais foi semelhante, apenas no D8 os grupos foram divididos nos tratamentos TCE8 e TBE9. De acordo com a Tabela 1, observa-se que já havia uma diferença no número de folículos dominantes no D8 para os animais selecionados aleatoriamente para o grupo TCE8, o que sugere que não foi o tratamento que influenciou os resultados de taxa de crescimento folicular e presença de ovulação. Certamente, os animais deveriam ter sido divididos nos dois tratamentos a partir da avaliação do tamanho do folículo dominante no D8 para que se separassem animais nas mesmas condições fisiológicas entre os tratamentos.

Como esperado, no dia 10 houve aumento no número de folículos dominantes $(>8 \mathrm{~mm})$ nos dois protocolos utilizados. Segundo Gimenes et al. (12) folículos subordinados cessam seu ritmo de crescimento entrando em atresia, enquanto o folículo dominante segue a uma taxa de crescimento constante, até alcançar a capacidade ovulatória. Assim, notou-se que as condições fisiológicas proporcionadas pelo protocolo de sincronização à base de estradiol e progesterona proporcionaram aumento do número de folículos em crescimento, como descrito por Bó et al. (13) isso é explicado por sincronizarem a emergência de uma nova onda folicular com êxito. Esses protocolos têm sido utilizados com sucesso para controlar a dinâmica folicular e luteal e para sincronizar a ovulação, permitindo IA sem a necessidade de detecção de estro em novilhas $(13,7,14)$.

Os resultados registrados para as taxas de crescimento folicular ( $\mathrm{mm} / \mathrm{dia})$, o diâmetro do folículo dominante no dia 10, intervalo da retirada do dispositivo de progesterona da IATF à ovulação e IATF à ovulação estão sumariados na Tabela 2. Não foi verificada diferença na taxa de crescimento folicular para o tratamento com cipionato de estradiol (CE) e benzoato de estradiol (BE) ( $>0,05$; Tabela 2). A taxa de crescimento folicular de 0,81 $\pm 0,63$ e $0,89 \pm$ $0,26 \mathrm{~mm}$ para $\mathrm{CE}$ e $\mathrm{BE}$, respectivamente, foi semelhante à registrada por Carvalho et al. (14) para novilhas B. taurus indicus, B. taurus indicus $x$ B. taurus taurus e B. taurus taurus, com a

Sobreira RR, Almeida IC, Oliveira FA, Siqueira JB, Barioni G, Lima DV, Siqueira LA. Cipionato de Estradiol e Benzoato de Estradiol em protocolos de inseminação artificial em tempo fixo em novilhas mestiças. Vet. e Zootec. 2017 Set.; 24(3): 581-591. 
utilização de BE no dia 9 como indutor de ovulação, sendo 0,$9 ; 1,2$; e 1,1 mm, respectivamente para as subspécies. Os valores verificados neste estudo aproximaram-se da taxa de crescimento em novilhas mestiças que não foram submetidas a protocolos de IATF, como os citados por Borges et al. (10), que oscilavam entre 1,0 a $1,4 \mathrm{~mm} / \mathrm{dia}$, valores que também se aproximam dos animais da raça Nelore tratadas com CE (0,9 mm/dia) (5).

Tabela 1. Média e desvio padrão do número de folículos de acordo com a classificação em folículos pequenos $(<6 \mathrm{~mm})$, folículos médios (entre $6-8 \mathrm{~mm})$ e folículos dominantes $(>8 \mathrm{~mm}$ ) em função dos tratamentos com $\mathrm{CE}$ e $\mathrm{BE}$ em protocolos de inseminação artificial em tempo fixo em novilhas mestiças.

\begin{tabular}{ccccc}
\hline & & \multicolumn{3}{c}{ Média do número de folículos } \\
\cline { 2 - 5 } & Dia & $<\mathbf{6 m m}$ & $\mathbf{6 ~ e ~ 8 m m}$ & $>\mathbf{8 m m}$ \\
\hline TCE8 & $\mathbf{0}$ & $10,0 \pm 3,13$ & $0,2 \pm 0,42$ & $0,6 \pm 0,52$ \\
$(\mathbf{n = 1 0})$ & $\mathbf{8}$ & $12,6 \pm 8,19$ & $0,1 \pm 0,32$ & $0,9 \pm 0,32^{\mathrm{a}}$ \\
& $\mathbf{1 0}$ & $7,4 \pm 2,5$ & $0,0 \pm 0,0$ & $1,0 \pm 0,00$ \\
TBE9 & $\mathbf{0}$ & $12,8 \pm 5,55$ & $0,5 \pm 0,53$ & $0,2 \pm 0,42$ \\
$(\mathbf{n}=\mathbf{1 0})$ & $\mathbf{8}$ & $15,2 \pm 6,82$ & $0,3 \pm 0,48$ & $0,2 \pm 0,42^{\mathrm{b}}$ \\
& $\mathbf{1 0}$ & $6,4 \pm 5,75$ & $0,2 \pm 0,42$ & $0,4 \pm 0,52$ \\
\hline
\end{tabular}

CE: cipionato de estradiol; BE: benzoato de estradiol; TCE8: tratamento com Cipionato de Estradiol aplicado no dia 8; TBE9: tratamento com benzoato de estradiol aplicado no dia 9. Médias seguidas por letras minúsculas diferentes, na mesma coluna, dentro do mesmo dia de tratamento, diferem entre $\operatorname{si}(\mathrm{P}<0,05)$

Tabela 2. Taxa de crescimento folicular, diâmetro do folículo dominante no D10, intervalo da retirada do dispositivo de progesterona da IATF à ovulação e IATF à ovulação (média \pm desvio-padrão), em função dos tratamentos com $\mathrm{CE}$ e $\mathrm{BE}$ em protocolos de inseminação artificial em tempo fixo em novilhas mestiças.

\begin{tabular}{|c|c|c|c|c|}
\hline \multirow[b]{2}{*}{ TRAT } & \multirow{2}{*}{$\begin{array}{l}\text { Tx. Cresc. Fol. } \\
(\mathbf{m m} / \text { dia })\end{array}$} & \multirow{2}{*}{$\begin{array}{c}\text { Diâm. } \\
\text { fol.dom. }(\mathrm{mm})\end{array}$} & \multicolumn{2}{|c|}{ Intervalo à ovulação* } \\
\hline & & & $\begin{array}{c}\text { Retirada do dispositivo de } \\
\text { progesterona (h) }\end{array}$ & IATF (h) \\
\hline TCE8 & $0,81 \pm 0,63$ & $10,97 \pm 1,24$ & $60,60 \pm 3,69^{\mathrm{a}}$ & $13,20 \pm 3,79$ \\
\hline TBE9 & $0,89 \pm 0,26$ & $9,77 \pm 1,36$ & $74,75 \pm 15,17^{\mathrm{b}}$ & $23,00 \pm 14,28$ \\
\hline
\end{tabular}

Médias seguidas por letras minúsculas diferentes, na mesma coluna, diferem entre si $(\mathrm{P}<0,05)$

As reduzidas taxas de crescimento $(\mathrm{mm} / \mathrm{dia})$ observadas nas novilhas do experimento estão possivelmente relacionadas às diferenças de padrões endocrinológicos de liberação de LH mediado pelas concentrações circulantes do dispositivo de $\mathrm{P}_{4}$.

Não houve diferença no diâmetro do folículo dominante no dia 10 para os tratamentos com CE e BE, conforme verificado na Tabela 2. As fêmeas tratadas com CE obtiveram um diâmetro de $10,97 \pm 1,24 \mathrm{~mm}$, enquanto as fêmeas submetidas ao tratamento com $\mathrm{BE}$ obtiveram um diâmetro de $9,77 \pm 1,36 \mathrm{~mm}$. Ferraz et al. (15), relacionaram folículos com maiores diâmetros ovulatórios a índices de concepção superiores.

Sá Filho et al. (16) em um experimento com novilhas Bos taurus indicus, que receberam $1 \mathrm{mg}$ de $\mathrm{CE}$ como indutor da ovulação, na retirada dos implantes de $\mathrm{P}_{4}$, obtiveram um folículo no dia 10 com o diâmetro de $11,3 \pm 0,4 \mathrm{~mm}$. Colazo et al. (7) trabalhando com novilhas mestiças registraram $11,3 \pm 2,1 \mathrm{~mm}$ no folículo dominante do dia 10, com aplicação de $1 \mathrm{mg}$ de $\mathrm{CE}$ concomitante a retirada da $\mathrm{P}_{4}$. Novamente, Colazo et al. (7) em seu experimento com 
novilhas mestiças avaliaram o diâmetro do folículo dominante, compararam novilhas tratadas com CE no dia $8(11,3 \pm 2,1 \mathrm{~mm})$, com novilhas tratadas com BE $24 \mathrm{~h}$ depois da retirada de $\mathrm{P}_{4}$ no dia $9(9,8 \pm 1,5 \mathrm{~mm})$, conseguindo resultados semelhantes ao do presente trabalho.

Borges e

$\mathrm{t}$ al. (10) estudaram o crescimento folicular ovariano sem o uso de indutores de ovulação, em 26 novilhas mestiças Holandês-Zebu, por meio de acompanhamento ultrassonográfico diário a partir do dia do estro e constataram que os diâmetros máximos dos folículos dominantes foram de aproximadamente $11,7 \mathrm{~mm}$, corroborando com os resultados referentes aos diâmetros para $\mathrm{CE}$ e $\mathrm{BE}$, deste experimento.

A utilização de estratégias buscando aumentar o diâmetro do folículo ovulatório são alternativas para melhorar a eficiência dos protocolos de sincronização. A mensuração do folículo pré-ovulatório, é considerada característica importante em protocolos de IATF, pois está relacionada ao tamanho do corpo lúteo e consequente produção de progesterona no diestro subsequente, essencial para a manutenção da gestação após a fertilização. Dessa forma, a taxa de prenhez em fêmeas submetidas à IATF pode ser comprometida na indução da ovulação de pequenos folículos, com formação de pequenos corpos lúteos e, por conseguinte, menor produção de progesterona, sendo que baixos níveis de progesterona circulante suprime o desenvolvimento embrionário inicial (7).

Sendo assim, a utilização de indutores de ovulação que resultem em folículos de maior tamanho, podem incrementar os resultados dos protocolos de sincronização.

Houve diferença no tempo (em horas) da retirada do dispositivo de progesterona à ovulação entre os tratamentos com CE e BE, conforme verificado na Tabela 2. As fêmeas tratadas com CE ovularam em média $60,60 \pm 3,69$ horas após a retirada do dispositivo de progesterona, enquanto as fêmeas submetidas ao tratamento com BE ovularam com $74,75 \pm 15,17$ horas após a retirada do dispositivo de progesterona.

O resultado do uso do CE se assemelham aos obtidos por Lopes et al. (17), no qual a ovulação em novilhas, ocorreu em média 60 a $62 \mathrm{~h}$ após a aplicação de CE. Martínez et al. (18) avaliaram 10 novilhas com o uso do BE $24 \mathrm{~h}$ como indutor de ovulação e conseguiram média de $72 \mathrm{~h}$ para esta variável, corroborando aos dados do presente trabalho (Tabela 2).

Todavia, em estudo realizado por Reis et al. (19), aponta que o uso do cipionato de estradiol no dia 8 em protocolos de IATF como indutor de ovulação em vacas nelore resulta em atraso nas ovulações (acima de $80 \mathrm{~h}$ ), sendo que estas ovulações não foram sincronizadas (80,0 $\pm 4,8 \mathrm{~h} ; 85,1 \pm 4,4 \mathrm{~h})$. Comparando ao estudo de Reis et al. (19), Martins et al. (20) em protocolos de IATF encontraram ovulações mais sincronizadas com o uso do BE no dia 9 $(67,4 \pm 1,69 \mathrm{~h})$ quando comparado ao CE no dia $8(76,8 \pm 2,82 \mathrm{~h})$. Fato este que não ocorreu no presente experimento.

No presente experimento, o tempo da retirada do dispositivo de progesterona à ovulação foi maior $(\mathrm{p}<0,05)$ para o tratamento com BE quando comparado ao CE (Tabela 2), observase que, numericamente, os animais do tratamento $\mathrm{CE}$ no dia 8 apresentaram folículos de $10,97 \pm 1,24 \mathrm{~mm}$ e ovularam mais cedo, enquanto as fêmeas tratadas com BE apresentaram folículos de menor diâmetro $(9,77 \pm 1,36 \mathrm{~mm})$ e ovularam mais tarde (Tabela 2). Segundo Gimenes et al. (12) quanto maior o tamanho do folículo, menor é o tempo para que estes folículos ovulem.

Não houve diferença quanto ao intervalo da inseminação artificial à ovulação $(p>0,05)$ (Tabela 2). As melhores taxas de concepção se concentram em inseminações realizadas entre 12 e $24 \mathrm{~h}$ antes da ovulação (16). Portanto, existe um intervalo de $12 \mathrm{~h}$ para que as inseminações sejam realizadas, sem que haja comprometimento dos índices reprodutivos (21). Verifica-se que mesmo com a variabilidade no tempo entre a IATF à ovulação, no presente estudo, os animais foram inseminados no momento propício, como mostra a tabela 2.

Sobreira RR, Almeida IC, Oliveira FA, Siqueira JB, Barioni G, Lima DV, Siqueira LA. Cipionato de Estradiol e Benzoato de Estradiol em protocolos de inseminação artificial em tempo fixo em novilhas mestiças. Vet. e Zootec. 2017 Set.; 24(3): 581-591. 
Os resultados para taxa de ovulação e taxa de prenhez para as novilhas mestiças submetidas aos tratamentos com CE e BE estão sumariados na Tabela 3. A taxa de ovulação dos animais tratados com BE foi de $40 \%(\mathrm{p}<0,05)$ sendo que, dos 10 animais avaliados, seis não responderam ao tratamento. As baixas taxas de ovulação observadas no presente estudo, para este tratamento, podem ser explicadas pela presença de folículos imaturos, incapazes de responder ao pico de LH induzido pela administração do BE. A ausência de crescimento destes folículos ocorre por uma escassez de receptores de LH como apontado por Gimenes et al. (12), ocorrendo atresia folicular e perda da capacidade ovulatória. Além disso, fatores relacionados à sensibilidade individual à progesterona liberadas pelos dispositivos, podem interferir sobre os resultados da ovulação, por exercer feedback negativo sobre aumento da frequência dos pulsos de LH (14).

Tabela 3. Taxa de ovulação (\%) e taxa de prenhez (\%) em função dos tratamentos com TCE8 e TBE9 em protocolos de inseminação artificial em tempo fixo em novilhas mestiças.

\begin{tabular}{ccc}
\hline Tratamento & Taxa de Ovulação $(\%)$ & Taxa de Prenhez $(\%)$ \\
\hline TCE8 & $90,00^{\mathrm{a}}$ & $37,03^{\mathrm{a}}$ \\
TBE9 & $40,00^{\mathrm{b}}$ & $37,03^{\mathrm{a}}$ \\
\hline
\end{tabular}

Houve diferença significativa na taxa de ovulação entre os tratamentos $(p<0,05)$ pelo teste de Qui-quadrado. Não houve diferença significativa na taxa de prenhez entre os tratamentos $(p>0,05)$ pelo teste de Qui-quadrado

No entanto, é importante ressaltar que, possivelmente, de acordo com a Tabela 1, observa-se que já havia uma diferença no número de folículos dominantes no D8 para os animais selecionados aleatoriamente para o grupo TCE8, o que sugere, que pode não ter sido o tratamento que influenciou o resultado de taxa de ovulação entre os grupos e sim, que já havia uma tendência dos animais do TCE8 em ovularem pela presença do maior número de folículos dominantes no D8.

Dados semelhantes para taxa de ovulação $(39,1 \%)$ foram registrados por Carvalho (22) que obtiveram em novilhas B. taurus indicus, utilizando BE como indutor de ovulação. Adicionalmente, Cavalieri et al. (23), trabalhando com novilhas cruzadas e Pegorer (24) com novilhas nelore, obtiveram taxa de ovulação de $69,6 \%$ e $65 \%$, respectivamente.

Em contraste, o aumento da taxa de ovulação nas novilhas do tratamento com $\mathrm{CE}$, de 90,0\% (Tabela 3) pode estar relacionada ao maior diâmetro do FD. A taxa de ovulação para $\mathrm{CE}$, foi similar às registradas por Rodrigues et al. (25) e Sá Filho et al. (16) que induziram a ovulação em novilhas nelore com CE e obtiveram uma taxa de 85\% e 81,5\%, respectivamente. Para Rodrigues et al. (25) o CE elevou as concentrações periféricas de estradiol, aumentando, por conseguinte o estímulo ovulatório.

A taxa de prenhez registrada no presente experimento para os dois tratamentos foi de $37,03 \%$. Não houve associação significativa entre tratamento e prenhez. Demonstrando assim que o tratamento não influenciou na prenhez. Meneghetti et al. (26) também não verificaram diferenças na taxa de prenhez entre vacas de corte que receberam cipionato de estradiol $(50,8 \%)$ concomitante a remoção do dispositivo de progesterona ou o benzoato de estradiol $(51,9 \%)$ aplicado $24 \mathrm{~h}$ depois da remoção do implante. Uslenghi et al. (27) não observaram diferenças na porcentagem de prenhez na IATF em novilhas, utilizando $1 \mathrm{mg}$ de CE (50\%) na retirada do dispositivo de $\mathrm{P}_{4}$, em comparação com a administração de $\mathrm{BE} 24 \mathrm{~h}$ depois (59\%).

Ainda, Chesta et al. (28), trabalhando com novilhas mestiças, obtiveram taxa de prenhez de $57,4 \%$ quando utilizaram $1 \mathrm{mg}$ de CE no D8 concomitante a retirada do dispositivo. Também, em novilhas holandesas, Chesta et al. (29) obtiveram 66,3\% e 54,4\% de prenhez utilizando $1 \mathrm{mg}$ de $\mathrm{CE}$ na retirada do dispositivo e BE $24 \mathrm{~h}$ depois, respectivamente, demonstrando diferença entre os tratamentos $(\mathrm{p}<0,05)$. 
Adicionalmente, os resultados obtidos no presente estudo se encontraram dentro da variação de prenhez, para a implantação de protocolos de IATF, que são de $23,6 \%$ à 74,4\% (13). Apesar desta larga margem, resultados que correspondem à taxa de prenhez acima da média não foram alcançados. No entanto, tem sido relatado que a sincronização do estro com a utilização do dispositivo de $\mathrm{P}_{4}$ resulta em uma baixa fertilidade no primeiro serviço (4). Além disso, não se deve descartar o efeito do clima, se tratando de um ano atípico, veranicos prolongados, início antecipado do período de seca e com a precipitação anual muito abaixo da média, $1190 \mathrm{~mm}$, o que podem ter prejudicado o desenvolvimento das pastagens e desempenho animal, assim, contribuíram para que não se alcançasse índices próximos de $50 \%$, que seriam ideais. Moreira (30) relatou que a restrição alimentar prolongada prejudicou a atividade cíclica das novilhas por suprimir a liberação do LH em pulsos de alta freqüência, necessários para o crescimento dos folículos ovarianos até o estágio pré-ovulatório.

Adicionalmente, o estresse também é um importante fator negativo que suprimi a secreção de LH (3), e Bos taurus indicus x Bos taurus taurus são mais temperamentais do que as raças taurinas (26). Motivo pelo qual, justifica a comparação de protocolos com diferentes indutores do estímulo ovulatório, no intuito de reduzir o trabalho e manejo com os animais. Dessa forma Colazo et al. (7) avaliaram os efeitos da utilização de CE no dia 8 e BE no dia 9 em novilhas de corte, e relataram que o tratamento com $\mathrm{CE}$ poderia ser realizado no momento da retirada do dispositivo de $\mathrm{P}_{4}$, e a IATF realizada $48 \mathrm{~h}$ mais tarde, reduzindo o manejo e stress, sem efeitos negativos sobre a taxa de prenhez $(63,33 \%, 63,1 \%$ para $\mathrm{CE}$ e $\mathrm{BE}$, respectivamente).

Contudo, a utilização de CE na retirada do dispositivo intravaginal, permite reduzir o número de manejo dos animais sem afetar a taxa de prenhez à IATF quando comparada ao $\mathrm{BE}$, em novilhas mestiças de corte ou de leite. Assim, o CE pode substituir o BE como indutor de ovulação em protocolos de IATF, reduzindo trabalho e custo.

\section{CONCLUSÃO}

A utilização do Cipionato de estradiol no dia 8, em protocolos de IATF em novilhas mestiças, resultou em menor tempo para a ovulação a partir da retirada do implante de progesterona;

Não houve diferença na taxa de prenhez de novilhas mestiças submetidas ao tratamento com cipionato de estradiol no dia 8 e benzoato de estradiol no dia 9 em protocolos de IATF;

A utilização do cipionato de estradiol comparado ao benzoato de estradiol é mais vantajosa visto que reduz o número de manejo, diminuindo o estresse dos animais e otimiza a mão de obra.

\section{COMITÊ DE ÉTICA E BIOSSEGURANÇA}

Este trabalho está de acordo com os princípios Éticos da Experimentação Animal, adotados pela Comissão de Ética no Uso de Animais (CEUA-UFES), sob o protocolo $\mathrm{n}^{\circ}$ $018 / 2014$.

\section{REFERÊNCIAS}

1. Oliveira JFC, Gonçalves PBD, Ferreira R, Gasperin B, Siqueira LC. Controle sobre GnRH durante o anestro pós-parto em bovinos. Cienc Rural. 2008;40:2623-31. 
2. Gottchall CS, Schuler MV, Martins CTDC, Almeida MR, Magero J, Soares JCR, et al. Efeitos do uso de GNRH no momento da IATF e dias pós-parto sobre a taxa de prenhez em vacas de corte com cria ao pé. Vet Foco. 2010;7:124-34.

3. Andrade BHA, Ferraz PA, Rodrigues AS, Loiola MVG, Chalhoub M, Ribeiro Filho AL. Eficiência do cipionato de estradiol e do benzoato de estradiol em protocolos de indução da ovulação sobre a dinâmica ovariana e taxa de concepção de fêmeas nelore inseminadas em diferentes momentos. Arch Vet Sci. 2012;17:70-82.

4. Torres JAP, Lópes JRA, Castro FGC, Monforte JGM. Comparación del cipionato de estradiol vs benzoato de estradiol sobre La respueta a estro y tasa de gestación em protocolos de sincronización com cidr em novillas y vacas Bos indicus. Univ Cienc. 2010;26:163-9.

5. Crepaldi GA. Eficácia de diferentes protocolos de indução da ovulação e de intervalos de inseminação em vacas de corte submetidas à IATF [dissertação]. São Paulo: Faculdade de Medicina Veterinária e Zootecnia, Universidade de São Paulo; 2009.

6. Palhão MP, Piedade CS, Araújo HL, Fernandes CAC, Guimarães CRB, Ribeiro JR, et al. Sincronização folicular e vascularização do folículo dominante em novilhas mestiças tratadas com estradiol. Rev Bras Cienc Vet. 2014;21:117-21.

7. Colazo MG, Kastelic JP, Mapletoft RJ. Effects of estradiol cypionate (ECP) on ovarian follicular dynamics, synchrony of ovulation, and fertility in CIDR-based, fixed-time AL programs in beef heifers. Theriogenology. 2003;60:855-65.

8. Baruselli PS, Reis EL, Marques MO, Nasser LF, Bó GA. The use of hormonal treatments to improve reproductive performance of anestrusbeef catlle in tropical climates. Anim Reprod Sci. 2004;82:486-97.

9. Vercesi Filho AE, Madalena FE, Albuquerque LG, Freitas AF, Borges LE, Ferreira JJ, et al. Parâmetros genéticos entre características de leite, de peso e a idade ao primeiro parto em gado mestiço leiteiro (Bos taurus x Bos indicus). Arq Bras Med Vet Zootec. 2007;59:983-90.

10. Borges ÁM, Torres CAA, Ruas JRM, Rocha Júnior VR, Carvalho GR. Dinâmica folicular ovariana em novilhas mestiças Holandês-Zebu. Arq Bras Med Vet Zootec. 2001;53:595-604.

11. SAS. Statistical Analysis System. SAS studio 3.2: administrator's guide. Cary: SAS; 2014.

12. Gimenes LU, Sá Filho MF, Carvalho NAT, Torres Junior JRS, Souza AH, Madureira $\mathrm{EH}$, et al. Follicle deviation and ovultory capacity in Bos indicus heifers. Theriogenology. 2008;69:852-8.

13. Bó GA, Baruselli PS, Martínez MF. Pattern and manipulation of follicular development in Bos indicus cattle. Anim Reprod Sci. 2003;78:307-26. 
14. Carvalho JBP, Carvalho NAT, Reis EL, Nichi M, Souza AH, Baruselli PS. Effect of early luteolysis in progesterone-based timed AI protocols in Bos indicus, Bos indicus x Bos taurus, and Bos taurus heifers. Theriogenology. 2008;69:167-75.

15. Ferraz PA, Rodrigues AS, Loiola MVG, Andrade BHA, Chalhoub M, Bittencourt RF, et al. Diâmetro do folículo pré-ovulatório no momento da inseminação artificial em tempo fixo em vacas nelore. In: Anais da XXIV Reunião Anual da Sociedade Brasileira de Tecnologia de Embriões; 2010; Porto de Galinha. Porto de Galinha: SBTE; 2010. p.81.

16. Sá Filho MF, Penteado L, Siqueira GR, Soares JG, Mendanha MF, Macedo GG, et al. Timed artificial insemination should be performed early when used norgestomet ear implants are applied for synchronizing ovulation in beef heifers. Theriogenology. 2013;80:642-7.

17. Lopes FL, Arnold DR, Williams J, Pancarci SM, Thatcher MJ, Drost M, et al. Use of estradiol cypionate for timed insemination. J Dairy Sci. 2000;83:214.

18. Martínez MF, Colazo MG, Kastelic JP, Mapletoft RJ. Effects o estradiol-17 $\beta$ or estradiol benzoate on follicular dynamics in CIDR-B-treated beef heifers. Theriogenology. 2002;57:382.

19. Reis EL, Gimenes LU, Marques MO, Carvalho JBP, Mapletoft RJ, Baruselli PS. Efeitos do cipionato e do benzoato de estradiol na dinâmica folicular e luteínica de vacas Nelore. In: Anais da XVIII Reunião Anual da Sociedade Brasileira de Tecnologia de Embriões; 2004; Barra Bonita. Barra Bonita: SBTE; 2004. p.236.

20. Martins CM, Castricini ESC, Sá Filho MF, Gimenes LU, Baruselli PS. Dinâmica folicular de vacas nelore tratadas com cipionato ou benzoato de estradiol em protocolos de inseminação artificial em tempo fixo. In: Anais da XIX Reunião Anual da Sociedade Brasileira de Tecnologia de Embriões; 2005; Angra dos Reis. Angra dos Reis: SBTE; 2005. p.285.

21. Roelofs JB, Graat EAM, Mullaart E, Soede NM, Voskamp-Harkema W, Kemp B. Effects of insemination-ovulation interval on fertilization rates and embryo characteristics in dairy cattle. Theriogenology. 2006;66:2173-81.

22. Carvalho JBP. Sincronização da ovulação com dispositivo intravaginal de progesterona (CIDR ${ }^{\circledR}$ ) em novilhas Bos indicus, Bos indicus x Bos taurus e Bos taurus [tese]. São Paulo: Faculdade de Medicina Veterinária e Zootecnia, Universidade de São Paulo; 2004.

23. Cavalieri J, Coleman C, Rodrigues H, Macmillan KL, Fitzpatrick LA. The effect of timing of administration of oestradiol benzoate on characteristics of oestrus, timing of ovulation and fertility in Bos indicus heifers synchronised with a progesterone releasing intravaginal insert. Aust Vet J. 2002;80:217-23.

24. Pegorer MF. Taxas de ovulação e prenhez em novilhas Nelore cíclicas após utilização de protocolos para inseminação artificial em tempo fixo (IATF), com diferentes concentrações de progesterona, associadas ou não a aplicação de ECG. [tese]. Botucatu: Faculdade de Medicina Veterinária e Zootecnia, Universidade Estadual Paulista; 2009.

Sobreira RR, Almeida IC, Oliveira FA, Siqueira JB, Barioni G, Lima DV, Siqueira LA. Cipionato de Estradiol e Benzoato de Estradiol em protocolos de inseminação artificial em tempo fixo em novilhas mestiças. Vet. e Zootec. 2017 Set.; 24(3): 581-591. 
25. Rodrigues ADP, Peres RFG, Lemes AP, Martins T, Pereira MMHC, Day ML, et al. Profesterone-based strategies to induce ovulation in prepubertal nellore heifers. Theriogenology. 2013;79:135-41.

26. Meneghetti M, Miguel JC. Addition of eCG on a fixed timed artificial insemination protocol in the conception rate of cycling nelore heifers. In: Anais da XXII Reunião Anual da Sociedade Brasileira de Tecnologia de Embriões; 2008; Guarujá. Guarujá: SBTE; 2008. p.638.

27. Uslenghi G, Chayer R, Callejas S. Efectividad del cipionato de estradiol injectado al final de um tratamiento com progesterona sobre la eficiência reproductiva. Rev Vet. 2010;21:55-8.

28. Chesta P, Brandan A, Cuestas G, Quiñones G, Lozano P, Tríbulo P. Evaluación de diferentes dosis de cipionato de estradiol sobre la tasa de preñez em inseminación artificial a tiempo fijo em vaquillonas de 15 meses de edad. In: Anais do VIII Simpósio Internacional de Reproducción Animal; 2009; Córdoba. Córdoba: Instituto de Reproducción Animal Córdoba; 2009. p.13.

29. Chesta P, Filippi L, Ramos M, Racca D, Bó G. Evaluación de las tasas de preñez em protocolos de inseminación artificial a tiempo fijo (IATF) utilizando diferentes dosis de cipionato de estradiol en vaquillonas holando. In: Anais do VIII Simpósio Internacional de Reproducción Animal; 2009; Córdoba. Córdoba: Instituto de Reproducción Animal Córdoba; 2009. p.12.

30. Moreira LDZ. Indução da puberdade em novilhas da raça nelore com progesterona veiculada em matriz polimérica [dissertação]. Campos dos Goytacazes: Universidade Estadual do Norte Fluminense; 2013.

Recebido em: 09/07/2016 Aceito em: 05/08/2017 